\title{
STARS IN THE GALACTIC HALO
}

\author{
T.D. KINMAN \\ Kitt Peak National Observatory \\ P.O. Box 26732, Tucson, AZ 85726, U.S.A. \\ (kinman@noao.edu)
}

\begin{abstract}
The field star halo is comprised of those field stars that are like the stars found in the halo globular clusters. We discuss the halo properties - in particular whether $\mathrm{V}_{\text {rot }}$ is a function of $z$. An analysis of recent surveys for blue horizontal branch stars is described; the halo that is defined by these stars is composite and contains both a spherical and flat component.
\end{abstract}

\section{Introduction}

At a symposium on stellar evolution held at the Eighth I.A.U., Walter Baade asked the simple question "Are we today in a position to point out two groups of stars, one which in terms of the cosmical time scale is young, the other which is old?" (Baade, 1954). He showed new color magnitude diagrams of M3 and M92, and explained why we think that globular clusters are old. Globular clusters not only contain the oldest stars that we know, they have one of the most extended distributions in the Galaxy and their stars are among those that show the least contamination by heavy elements - the products of stellar nucleosynthesis. Not all globular clusters have exactly the same age and not all belong to the halo. The disk globulars are a younger subset; the distinction between them and the halo globular clusters, that define Population II, is summarized in Table 1 (Zinn, 1985; Armandroff, 1989).

In this paper, we define the field star halo as those field stars that are like the stars found in the halo globular clusters. In particular, they are to be distinguished from the field stars that belong to the "thick disk" ${ }^{1}$ Even

\footnotetext{
${ }^{1}$ A loose connection clearly exists between the disk globular clusters and the field star population variously called "the thick disk", "the extended disk", and "Intermediate 
TABLE 1. Properties of Disk and Halo Globular Clusters.

\begin{tabular}{lll}
\hline Property & Halo Clusters & Disk Clusters \\
\hline Galactic Distribution & Spherical & Flat, Scale height 0.8 to $1.5 \mathrm{kpc}$ \\
Metallicity & {$[\mathrm{Fe} / \mathrm{H}]<-0.8$} & {$[\mathrm{Fe} / \mathrm{H}]>-0.8$} \\
Galactic Rotation & $+50 \pm 23 \mathrm{~km} / \mathrm{s}$ & $+193 \pm 29$ \\
Horizontal Branch & BHB, RR Lyrae \& RHB & Only RHB \\
\hline
\end{tabular}

though the halo globulars have a large spread in some of their properties (e.g. metallicity), there are enough halo clusters to allow the distribution functions of such properties to be to be quite well determined; it is therefore practical to use the properties of the halo globular stars to define the field star halo. The classification of individual stars may be problematic if the criteria are apparently conflicting. Thus, on our definition, the high-velocity, very metal-poor $([\mathrm{Fe} / \mathrm{H}]=-3.1)$ spectroscopic binary CS 22873-139 would not be said to belong to the field star halo because it is too young ( $8 \mathrm{Gyr}$; Preston , 1994) even though on a simple definition in terms of metallicity and kinematics we might call it a halo star. The existence of stars such as CS 22873-139 makes the problem of identifying halo stars non-trivial and challenging.

Near the Sun, "thick disk" stars outnumber halo stars by perhaps 10 to 1 , and so any local halo sample may well contain "thick disk" stars unless quite sharp criteria are applied. The best halo tracers are therefore stars which have the lowest probability of being confused with those of some other more dominant population; the purity of a sample will be highest when its constituents satisfy as many of the defining properties as possible. Variables make good tracers because the extra observables associated with their variabilty usually allow a precise classification to be made. Late type giants that have been selected by color make poor tracers; it has been said (Hoyle, 1965) that "the giant sequence is a shelf that the stars sit on almost irrespective of their other properties".

\section{RR Lyrae Stars as Tracers of the Halo}

Large numbers of RR Lyrae stars were discovered in the the early surveys for variable stars at Harvard, Leiden and Sonneberg. The widespread discovery of these stars at high galactic latitudes was the first sign that a

Population II" (nomenclature discussed by Majewski, 1993). The "thick disk" population is too complex, however, to be described as consisting of stars like those found in the disk globular clusters. 
field star halo exists. This early work is described in IAU Symposium No 1 (Blaauw, 1955; held in Groningen in 1954) where the words "halo", "disk" and "nucleus" were first used to indicate parts of the Galaxy ${ }^{2}$ At this Symposium Baade proposed the Palomar-Groningen Survey for variable stars in order to determine "the surfaces of equal density of the halo"; for details see Oort et al. (1975) and Wesselink et al. (1987).

The field RR Lyrae stars of the same period range as those in globular clusters have similar galactic kinematics to the clusters (Kinman , 1959; Preston , 1959) and also a similar metallicity range; they could also be easily recognized at large distances with the photographic detectors that were available thirty years ago. This led me to start a survey with the Lick Astrograph that was specifically for RR Lyrae stars (Kinman et al., 1965; Kinman et al., 1966; Kinman et al., 1982) that is still being continued. This and other surveys for fainter RR Lyrae stars (Hawkins, 1984; Saha, 1985) give a picture of the halo that is spheroidal $(\mathrm{c} / \mathrm{a} \sim 0.6)$ inside the solar circle and more or less spherical at larger galactocentric distances $\mathrm{R}_{\text {gal }}$ (Hartwick, 1987; Wesselink et al., 1987). The space-density of these stars falls off with an inverse power law whose exponent has been estimated between -3.0 and -3.5; a more rapid fall-off with exponent -5.0 for $\mathrm{R}_{\text {gal }}>25 \mathrm{kpc}$ is possible (Saha, 1985) . A similar space-density exponent is found for the halo globular clusters (Harris, 1976; Zinn, 1985).

Abundance studies (Suntzeff et al., 1991), show that outside the solar circle the RR Lyraes have the same abundance distribution as the globular clusters and (like them) show no abundance gradient with either $z$ or $R_{\text {gal }}$; a more complex situation exists within the solar circle. It has been estimated (Kinman , 1992) that the RR Lyrae stars observed in the Lick survey represent all but the most metal-poor $20 \%$ of the halo population. Despite the wide variety of HB morphologies found in halo globular cluster$\mathrm{s}$, which has caused doubt as to the value of RR Lyrae stars as halo tracers (c.f.Majewski, 1993), these stars have provided, until quite recently, most of our information about the structure of the field star halo.

\section{The Halo from Spectroscopic and Multicolor Surveys}

Much recent work has been devoted to the study of low metal abundance stars in the solar neighbourhood. Primary sources for this work include specific objective-prism surveys for metal-weak stars (Bond, 1970; Bond, 1980; Ratnatunga et al., 1985; Ratnatunga et al., 1989) source lists (Bidelman et al., 1973) and follow-up spectroscopy and photometry (Norris et al., 1985). The non-kinematically selected metal-weak stars derived from these

\footnotetext{
${ }^{2}$ There still seems little agreement as to whether the word "halo" should be used to describe a space or, as here, a stellar population.
} 
sources are assigned to populations by metallicity and kinematic properties. Others have used wide field multicolor surveys to identify the stars of different populations by their colors. The RGU surveys by the Basel group (Becker, 1965; Buser et al., 1994) and the broad-band surveys with the UK Schmidt (Reidet al., 1982; Gilmore, 1984) pioneered this approach. A detailed review of these various methods has recently been given (Majewski, 1993).

How well do the galactic rotations of the stars in these various halo samples agree with the galactic rotation of the halo globular clusters (Table $1)$ ? Table 2 gives this $\mathrm{V}_{\text {rot }}$ for a number of recent surveys - roughly in order of increasing height $z$ of the halo sample above the galactic plane.

TABLE 2. Determinations of the Halo Rotation $\mathrm{V}_{\text {rot }}$ in $\mathrm{km} / \mathrm{s}$.

\begin{tabular}{llll}
\hline Location of Survey & Metallicity Limit & $\mathrm{V}_{\text {rot }}$ & Reference \\
\hline Solar Neighborhood & {$[\mathrm{Fe} / \mathrm{H}] \leq-1.5$} & $+09 \pm 07$ & Carney et al. $(1986)$ \\
Solar Neighborhood & {$[\mathrm{Fe} / \mathrm{H}] \leq-1.2$} & $+37 \pm 10$ & Norris (1986) \\
Solar Neighborhood & {$[\mathrm{Fe} / \mathrm{H}] \leq-1.6$} & $+25 \pm 15$ & Morrison et al. $(1990)$ \\
Solar Neighborhood & {$[\mathrm{Fe} / \mathrm{H}] \leq-1.5$} & $+30 \pm 10$ & Ryan et al. $(1991)$ \\
$\mathrm{Z}=1$ to $2 \mathrm{kpc}$ & & $+58 \pm 12$ & Soubiran $(1993)$ \\
$\mathrm{Z}=1$ to $2 \mathrm{kpc}$ & & -08 & Karaali $(1994)$ \\
$\mathrm{Z}=1$ to $2 \mathrm{kpc}$ & & $+87 \pm 09$ & Ojha et al. $(1994)$ \\
North Galactic Pole & & $-20 \pm 30$ & Reid (1990) \\
$\mathrm{Z} \geq 5 \mathrm{kpc}$ & & $-47 \pm 09$ & Majewski (1992) \\
\hline
\end{tabular}

The samples near the plane ${ }^{3}$ give (with some scatter) a $V_{\text {rot }}$ close to that derived for the halo globular clusters. The last two surveys that are well out of the plane give a retrograde $\mathrm{V}_{\text {rot }}$.

Allen, Schuster and Poveda have derived galactic orbits for a sample of very metal-weak $([\mathrm{Fe} / \mathrm{H}] \leq-2.0)$ stars in the solar neighbourhood (Allen et $a l ., 1991)$. The mean $\mathrm{V}_{\text {rot }}$ of these stars is $+11 \pm 19 \mathrm{~km} / \mathrm{s}$ which is in good agreement with other determinations for the solar neighbourhood (Table 2). If, however, we take the 10 stars in their sample whose $\left|z_{\text {max }}\right|$ exceeds 10 $\mathrm{kpc}$, we find that their mean $\mathrm{V}_{\text {rot }}$ is $-92 \pm \mathrm{km} / \mathrm{s}$, while the 24 stars whose $\left|\mathrm{z}_{\text {max }}\right|$ is less than $2.5 \mathrm{kpc}$ have a mean $\mathrm{V}_{\text {rot }}$ of $+71 \pm 20 \mathrm{~km} / \mathrm{s}$. This gives independent support for the conclusion that halo stars far from the plane tend to have more retrograde orbits. If we take only those stars in the Allen et al. sample that have $+4.5 \leq \mathrm{M}_{v} \leq+6.7$ and $0.24 \leq(\mathrm{b}-\mathrm{y})_{0} \leq+0.40$ (i.e. just the subdwarfs), the 8 stars with $\left|z_{\text {max }}\right|$ greater than $10 \mathrm{kpc}$ have a mean

\footnotetext{
${ }^{3}$ Including a small sample of local BHB stars (Kinman et al., 1994)
} 
$\mathrm{V}_{\text {rot }}$ of $-76 \pm 41 \mathrm{~km} / \mathrm{s}$ and the 14 stars whose $\left|z_{\text {max }}\right|$ is less than $2.5 \mathrm{kpc}$ have a mean $\mathrm{V}_{\text {rot }}$ of $+46 \pm 22 \mathrm{~km} / \mathrm{s}$. Again, this suggests that the $\mathrm{V}_{\text {rot }}$ of halo field stars is a function of $z$ at the solar galactocentric distance ${ }^{4}$. As an explanation, we might consider the possibility that the halo samples of stars that stay near the plane are significantly adulterated by very low metallicity disk stars. But if this is true, why is the $\mathrm{V}_{\text {rot }}$ of the solar neighborhood halo similar to that of the halo globular clusters?

\section{The Halo Defined by the Blue Horizontal Branch (BHB) Stars.}

The arguments against using RR Lyrae stars as halo tracers have been (a) the uncertainty in how representative they are because of the variable morphology of the horizontal branch, and (b) the observational problem of detecting low-amplitude RR Lyrae variables by traditional techniques. Both arguments can be met (Kinman, Suntzeff and Kraft 1994) by using an objective-prism survey for AF stars as the source of both the BHB stars and RR Lyrae stars since these two groups together effectively define the HB type ${ }^{5}$. The stars of spectral type A and F (AF stars) were obtained by the late Nick Sanduleak as part of the Case Low-Dispersion Northern Sky Survey (Pesch et al., 1983; Sanduleak, 1988) for two fields (SA 57 at the NGP and the Anticenter field RR 7) that had already been searched for RR Lyrae stars with the Lick Astrograph. The AF survey was shown to be complete in the magnitude range $13 \leq \mathrm{V} \leq 16.5$. The $\mathrm{RR}$ Lyrae stars (all amplitudes) were found by photoelectric monitoring of the AF stars; in addition to the higher-amplitude RR Lyrae stars previously found in the Lick survey, we discovered a roughly equal number of lower-amplitude variables.

The BHB stars were isolated in three ways. First, by using their $(u-B)$ color (Stromgren $\mathrm{u}$ and Johnson B) in a method similar to the use of the Stromgren $c_{1}$ index (Philip et al., 1994) which measures the size of the Balmer Jump. The second was to use the $\mathrm{D}_{0.2}$ width of the Balmer lines

\footnotetext{
${ }^{4} \mathrm{It}$ is clearly of interest to know if these two groups of subdwarfs also have different physical properties. For those interested in investigating this possibility, the Hipparchos Input Catalogue (HIC) numbers for the subdwarfs whose $\left|\mathrm{z}_{\max }\right|$ exceeds $10 \mathrm{kpc}$ are: 36513 (10.8); 46516 (11.1); 47480 (10.5); 48152 (8.3); 66673 (11.5); 68321 (10.0); 68592 (11.1) and 86458 (9.9). The figures in parentheses are V magnitudes. The HIC numbers for the subdwarfs whose $\left|\mathrm{z}_{\max }\right|$ is less than $2.5 \mathrm{kpc}$ are: $8572(10.4) ; 12807(11.4) ; 15396$ (11.2); 36430 (10.4); 48195 (11.0); 59109 (10.0); 72461 (9.7); 76976 (7.2); 87693 (9.9); 91129 (11.4); 96099 (10.2); 96115 (9.4); 106468 (10.6) and 111372 (9.7).

${ }^{5}$ In principle the red HB component should also be known, but a test using the $B$, $\mathrm{V}$ and $\mathrm{R}$ (i.e. BHB, RR Lyrae and RHB) counts for globular clusters (Lee et al., 1994), showed that $B-V /(B+V)$ correlated well with $B-R /(B+V+R)$ for the more populous halo clusters except NGC 2808.
} 
$\mathrm{H} \gamma$ and $\mathrm{H} \delta$ (Searle et al., 1966). The third was to use a parameter $\Lambda$ which measures the steepness of the Balmer jump ${ }^{6}$ The three methods were calibrated using both local BHB stars and also BHB stars in the halo globular clusters $\mathrm{M} 3$ and $\mathrm{M} 92$ and gave consistent results in the color range $0.00 \leq(\mathrm{B}-\mathrm{V})_{0} \leq+0.20$. For the AF stars with $\mathrm{B}>13$, about half those in SA 57 at the NGP and about one third of those in the Anticenter field (b $=+37^{\circ}$ ) were BHB stars. Metallicities ${ }^{7}$ of these BHB stars had a similar distribution to that of the RR Lyrae stars in the same fields and to that of the halo globular clusters outside the solar circle.

Space densities were derived for these BHB stars and also from earlier observations of field BHB stars (Arnold et al., 1992; Sommer-Larsen et al., 1989; Preston et al., 1991). Only the high-latitude data from these other surveys were used and some corrections were applied to them to allow for the inclusion of non-BHB stars. The space densities derived from the stars with $z>5$ kpc show a classical spherical halo which follows a $\mathrm{R}_{\text {gal }}^{-3.5}$ law with an HB morphology like that of the globular cluster M3 (roughly equal numbers of BHB and RR Lyrae stars). In analyzing the space densities nearer the plane, the local space density of BHB stars (Green et al., 1993) was also used. In this region an additional flatter component was found. The analysis of Kinman et al. gave this component a scale height of 2.2 $\mathrm{kpc}$; the inclusion of more recent data suggests that the scale height of the flat component is closer to $1.5 \mathrm{kpc}$. The stars of the two components do not have significantly different metallicity distributions but do have slightly different mean values of $\Lambda$ (the measure of the steepness of the Balmer jump). Also, if present estimates of the local RR Lyrae space density are correct, the flat component (which makes up $80 \%$ of the BHB stars in the solar neighborhood) has a bluer HB morphology than the spherical component. The total numbers of these two BHB halo populations are rather comparable. ${ }^{8}$

Our knowledge of the structure of the two halo components is still rather imprecise because the number of properly identified BHB stars is still small. This should be remedied when the extension of the Kinman et al. survey is completed and sharp criteria applied to the candidates in other substantial new surveys (Rodgers et al., 1993; Beers et al., 1994).

This composite model of the halo based on observations outside the solar circle complements and agrees with an earlier analysis (Hartwick,

\footnotetext{
${ }^{6} \Lambda$ was determined using precepts similar to those used in deriving $\lambda_{1}$ in the BCD system (Chalonge et al., 1973).

${ }^{7}$ these were primarily derived from the Mg II $\lambda 4481 \AA$ and Ca II $\lambda 3933 \AA$ lines and calibrated by local and cluster BHB of known metallicity.

${ }^{8}$ Integrating the spherical component through the range $6 \leq R_{g a l} \leq 20 \mathrm{kpc}$ gives a total count of $\sim 50,000$ stars. Integrating the infinite cylinder containing the flat component between radii with $R_{\text {gal }}=6$ and $R_{\text {gal }}=20 \mathrm{kpc}$ gives a total count of $\sim 100,000$ stars.
} 
1987) of the RR Lyrae stars inside the solar circle. Further support for this composite model comes from a recent analysis of 280 local halo stars (Schuster et al., 1994). The distribution of $\left|z_{\max }\right|$ for these stars (given in their Fig. 6) shows that $63 \%$ of their sample have $\left|\mathrm{z}_{\max }\right| \leq 2.5 \mathrm{kpc}$ (i.e. a flat component), while the remainder have $2.5 \leq\left|\mathrm{z}_{\max }\right| \leq 15.0 \mathrm{kpc}$ (i.e. a more spherical component). Finally, the ratio of the local density of halo giants to the local density of $\mathrm{BHB}$ stars is similar to the ratio found in the halo globular clusters (Morrison, 1993). This supports the conclusion that the composite model that was found for the BHB stars has a general application to all halo field stars.

\section{Conclusions and Questions}

The halo is not homogeneous. Besides the distinction between an inner and an outer halo (Searle et al., 1978), the field star halo beyond the solar circle shows structure as a function of $z$. In addition to the spherical halo there is a flatter halo whose properties (the presence of BHB stars and low $V_{\text {rot }}$ ) distinguish it from a metal-poor "thick disk". In connection with this model, it is important to confirm if and how $\mathrm{V}_{\text {rot }}$ varies with $z$. The globular clusters that have retrograde motions have been said to have specific physical properties (Rodgers et al., 1984; van den Berg, 1993); does a similar effect exist among the field halo stars? More generally, are there specific physical differences between the stars of the two halo components that would help us investigate the processes of their formation?

\section{References}

Allen, C., Schuster, W. J. and Poveda, A., 1991, A. \& A. 244, 280

Armandroff, T. E., 1989, A. J. 97, 375

Arnold, R. and Gilmore, G., 1992, M. N. R. A. S. 257, 225

Baade, W., 1954, Trans. IAU VIII, 682

Becker, W., 1965, Z. Ap. 62, 54

Beers, T.C. and Wilhelm, R., 1994, Hot Stars in the Halo, eds. S. Adelman and A. Upgren, Cambridge University Press, (in press)

Bidelman, W. P. and MacConnell, D. J., 1973, A. J. 78, 687

Blaauw, A., 1955, Co-ordination of Galactic Research, I.A.U. Symp. 1, Cambridge University Press, p. 4

Bond, H. E., 1970, Ap. J. Suppl. 22, 117

Bond, H. E., 1980, Ap. J. Suppl. 44, 517.

Buser, R. and Rong, J., 1994, Unsolved Problems of the Milky Way, I.A.U. Symp. 169, preprint

Carney B. W. and Latham, D. W., 1986, A. J. 92, 60

Chalonge, D. and Divan, L., 1973, A. \& A. 23, 69

Gilmore, G., 1984) M. N.R.A.S. 207, 223

Green, E. M. and Morrison, H. L., 1993, The Globular Cluster - Galaxy Connection, ASP Conf. Ser. 48, eds. G. Smith and J. Brodie, ASP San Francisco, p. 318

Harris, W. E., 1976, A. J. 81, 1095 
Hartwick, F. D. A., 1987, The Galaxy, eds. G. Gilmore and B. Carswell, D. Reidel, Dordrecht, p. 281

Hawkins, M. R. S., 1984, M. N. R. A. S. 206, 433

Hoyle, F., 1965, Roy. Obs. Bull. 82, E53

Karaali, S., 1994, A. \& A. Suppl. 106, 107

Kinman, T.D., 1959, M. N. R. A. S.,119, 559

Kinman, T.D., 1992, Elements and the Cosmos, eds. M. Edmunds and R. Terlevich, Cambridge University Press, p. 151

Kinman, T. D., Wirtanen, C. A. and Janes K. A., 1965, Ap. J. Suppl. 11, 223

Kinman, T. D., Wirtanen, C. A. and Janes K. A., 1966, Ap. J. Suppl. 13, 379

Kinman, T. D., Mahaffey, C.T. and Wirtanen, C. A., 1982, A. J. 87, 314

Kinman, T. D., Suntzeff, N. B. and Kraft, R. P., 1994, A. J., in press

Lee, Y.-W., Demarque, P. and Zinn, R., 1994, Ap. J. 423, 248

Majewski, S. R., 1992, Ap. J. Suppl. 78, 87

Majewski, S. R., 1993, Ann. Rev. Astron. Astrophys. 31, 571

Mathieu, R. D. and Mazeh, T., 1988, Ap. J. 326, 256

Morrison, H. L. Flynn, C. and Freeman, K. C., 1990, A. J. 100, 1191

Morrison, H. L., 1993, A. J. 106, 578

Norris, J., Bessell, M. S. and Pickles, A. J., 1985, Ap. J. Suppl. 58, 463

Norris, J., 1986, Ap. J. Suppl. 61, 667

Ojha, D. K., Bienhamé, O., Robin, A. C. and Mahan, V., 1994, A. \& A., submitted

Oort, J. H. and Plaut, L., 1975, A. \& A. 41, 71

Pesch, P. and Sanduleak, N., 1983, Ap. J. Suppl. 51, 171

Philip, A. G. D. and Adelman, S. J., 1994, Workshop on Databases for Galactic Structure, eds. A. Philip, B. Hauck and A. Upgren, Davis Press, Schenectady, p. 245

Preston, G. W., 1959, Ap. J. 130, 507

Preston, G. W., 1994, preprint

Preston, G. W., Shectman, S. A. and Beers, T. C., 1991, Ap. J. 375, 507

Ratnatunga, R. U. and Freeman, K. C., 1985, Ap. J. 291, 260

Ratnatunga, R. U. and Freeman, K. C., 1989, Ap. J. 339, 126

Reid, N. and Gilmore, G., 1982, M. N. R. A.S. 201, 73

Reid, N., 1990, M. N.R. A. S. 247, 70

Rodgers, A. W. and Paltoglou, G., 1984, Ap. J. 283, L5

Rodgers, A. W. and Roberts, W. H., 1993, A. J. 106, 2294

Ryan, S. G., Norris, J. E., 1991, A. J. 101, 1835

Saha, A., 1985, Ap. J. 289, 310

Sanduleak, N., 1988, Ap. J. Suppl. 66, 309

Schuster, W. J. and Allen, C., 1994, Unsolved Problems of the Milky Way, IAU Symp. 169 , preprint

Searle, L. and Rodgers, A.W., 1966, Ap. J. 143, 809

Searle, L. and Zinn, R., 1978, Ap. J. 225, 357

Sommer-Larsen, J., Christensen, P. and Carter, D., 1989, M. N. R. A. S. 238, 225

Soubiran, C., 1993, A. \& A. 274, 181

Suntzeff, N. B., Kinman, T. D. and Kraft, R. P., 1991, Ap. J. 367, 528

van den Berg, S., 1993, A. J. 105, 971

Wesselink T. H., Le Poole, R. S. and Lub. J., 1987, Stellar Evolution and Dynamics in the Outer Halo of the Galaxy, eds. M. Azzopardi and F. Matteucci, (E.S.O., Garching), p. 187

Zinn, R., 1985, Ap. J. 293, 423

ROMAN: On your list of stars at low $\mathrm{z}$ with prograde rotation and at high $\mathrm{z}$ with retrograde rotation, it was striking that the high-z stars were in a very limited range of Right Ascensions. Cor:ld this have affected you:r rest:lt?

KINMAN: I identified the stars by their HIPPARCHOS Inpt:t Catalog nt:mbers. 
Actc:ally, the high-z stars with retrograde rotation range in RA from 7 to 17 hot.rs. KRAFT: Do yor: know how we might identify the giants and st.bdwarfs that correspond to the two poptlations of halo blte HB stars?

KINMAN: The halo giants and stbdwarfs are most easily classified by their orbits - in partictlar by their $\left|Z_{\max }\right|$.

TAYLER: Can yot: associate a number of globtlar cltsters with each of yotr field halo components?

KINMAN: I have not yet been able to do this.

KING: With regard to the absence of your blte stars in disk globtlars, we shotld remember the low $-\Delta S$ RR Lyraes, which have the metallicity and kinematics of disk globtlars, brtt are not seen in those cltsters. Prestmably, this is a smallntmber effect; might this not also be the case for your blte stars? At first I was strick that yotr "flat halo" grot:p has the spatial distribttion of the thick disk, brt a lower metallicity. Br.t now I wonder if they aren't also part of the thick-disk poptlation, brt as rare as not to be seen in the globtlars.

KINMAN: The flat halo BHB stars as well as being absent in the disk globtlar clisters are distingtished from the "thick disk" by having a low galactic rotation. 